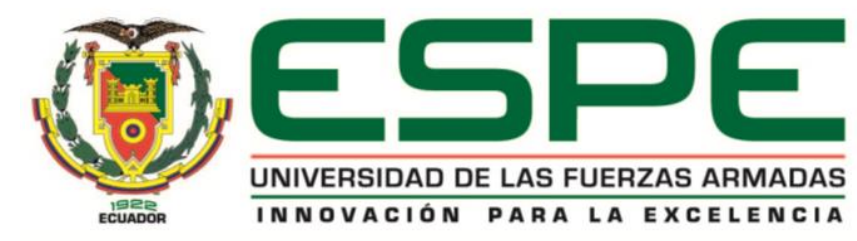

\title{
Control of an Omnidirectional Robot Based on the Kinematic and Dynamic Model
}

\author{
Gallo Jiménez, Luis Vinicio y Paste Guanoluisa, Byron Paste \\ Departamento de Eléctrica y Electrónica \\ Carrera de Ingeniería en Electrónica e Instrumentación
}

Artículo académico, previo a la obtención del título de Ingeniero en Electrónica e Instrumentación

Ing. Andaluz Ortiz, Víctor Hugo, PhD.

5 de julio del 2021 


\title{
Control of an Omnidirectional Robot Based on the Kinematic and Dynamic Model
}

\author{
Luis V. Gallo ${ }^{(\bowtie)}$, Byron D. Paste ${ }^{(\bowtie)}$, Jessica S. Ortiz ${ }^{(\bowtie)}$, \\ and Víctor H. Andaluz ${ }^{(凶)}$ \\ Universidad de las Fuerzas Armadas ESPE, Sangolquí, Ecuador \\ \{lvgallo, bdpaste, jsortiz4,vhandaluzl\}@espe.edu.ec
}

\begin{abstract}
This work focuses on the proposal for the implementation and evaluation of control algorithms for the monitoring of autonomous trajectories based on the kinematic and dynamic model of an omnidirectional robotic platform in four wheel configuration type mecanum also built for the process of identification and validation of the dynamic parameters obtained from the mathematical model by means of an identification algorithm, the evaluation of these control algorithms is carried out experimentally on the Robotic Platform in real four-wheel omnidirectional configuration with which it also allows to evaluate the operation of the Hardware in the Loop control scheme (HIL), a technique that constitutes the untimely connection of external hardware with computer equipment allowing to simulate in real time the behavior of the robotic system with omnidirectional traction in four-wheel configuration type mecanum. Subsequently the data resulting from the Hardware in the Loop (HIL) control scheme will be compared to the data obtained in the experimental test for corresponding validation.
\end{abstract}

Keywords: Omnidirectional robot $\cdot$ Mecanum wheel $\cdot$ Hardware-in-the-Loop

\section{Introduction}

In recent years, the robotics industry has developed rapidly. A lot of technologies are implemented in various applications [1,2], e.g., mobile robots have the ability to perform specific tasks in an industrial work environment, homes, hospitals, among others [3]. Some representative mobile robots include household cleaning robots, military surveillance drones, warehouse robots and autonomous robots [4, 5]. The latter have become indispensable components in many applications, including research, warehouse management, surveillance and safety, and autonomous vehicles [6, 7].

Currently there are different types of platforms that are characterized by the type of wheels or by the mechanical structure that makes up it among the main ones can be detailed: i) Unicycle robots, they are formed of a mechanical structure with three degrees of freedom consisting of two conventional fixed wheels and a stable partial wheel that is independently controlled on the same axle, so it can automatically navigate in a specific work environment. This type of robot is the most commonly used in security, surveillance, transport, education and research applications [8]; ii) robots carlike, it has its kinematic model based on Ackerman's mobility system with its linear 
speed and rotation angle, this model has a fairly large studio and is the basis for the current car [9]. Its use has increased significantly due to advances in sensors and control systems, these range from Google cars to modern automatically guided vehicles. They are used in industrial applications to transport materials in manufacturing, value-added and warehousing operations [10]; and iii) omnidirectional robots, A vehicle that can drive with 3 degrees of freedom in a two dimensional space and also independently control, translation and rotation can be considered as an omnidirectional robot [11]. By configuration, the omnidirectional robot can move in the desired direction from any starting position [12], because the mobile system ensures perfect mobility in any narrow space (curved, diagonal or any other space) because of its type of mecanum wheels [13]. Due to their versatility they can perform the functions of suppliers, inspectors, supervise different tasks in remote or explosive areas and provide support or bases for other robots or manipulators, because of all these features the demand for the use of omnidirectional robots has increased [12].

Among the different works carried out with omnidirectional robots we can detail: $i$ ) construction, the theory describes that for the construction of such omnidirectional robots with mecanum wheel the $\mathrm{AB}$ configuration is more used [12, 14], and the arrangement of the wheels are made rectangular or in turn commercial type robots such as KUKA Youtbot are used $[15,16]$; ii) modeling, the previously designed models employ only the robot's kinematics regardless of the advantages provided by the dynamic model for controller stability and when the dynamic model is presented it has as inputs torques or voltages as shown in [17], where as a contribution to the dynamic model the dynamics of the engines were considered in this case the system inputs are the armature voltages of each engine; iii) implementing control algorithms, Several of the articles use nonlinear controllers based on linear algebra, numerical or unifiedbased methods in the robot's kinematics with dynamic compensation, e.g., [18], it details the implementation of a kinematic controller with internal dynamic compensation that reduces the speed tracking error.

One of the problems that exists in the field of robotics, is directly being able to experience control algorithms in which the necessary hardware is not available because of its high cost or its limitation for the number of tests, for which one of the alternatives for industrial automation processes is hardware in the loop (HIL). This is a technique in which a real time simulation environment is developed to test the behavior of its control algorithms without physical prototypes $[19,20]$. It is widely used in the automotive industry to test vehicle dynamics and drivers [21], also used in aerospace and industrial automation. Therefore, this work proposes the implementation of the hardware in the loop technique in a virtual environment in real time, to evaluate control algorithms based on the kinematic and dynamic model of an omnidirectional robotic platform in four wheel configuration type mecanum, using as inputs speeds with what differs from previous jobs $[12,14,17]$, will also be validated through the implementation of the control algorithm in a real omnidirectional robot.

The article is organized as follows: Sect. 2 presents the kinematic and dynamic modeling of the four-wheel omnidirectional mecanum robot; the control scheme of the HIL, control algorithm design and stability analysis are presented in Sect. 3; Experimental results are presented and discussed in Sect. 4. Finally, the conclusions are given in Sect. 5. 


\section{Robot Omnidirectional}

Among the fundamental parts in the design of controllers for robotic systems are based on obtaining mathematical models that represent the characteristics and restrictions of movement, from a cinematic and dynamic point of view; therefore, this section presents the obtaining of the Kinematic Model and the Dynamic Model of the omnidirectional robotic platform, in which two linear speeds and an angular speed are considered as signs of maneuverability.

\subsection{Kinematic Model}

Obtaining this model involves analyzing the positions and speeds required to perform autonomous tasks such as path tracking (Fig. 1).

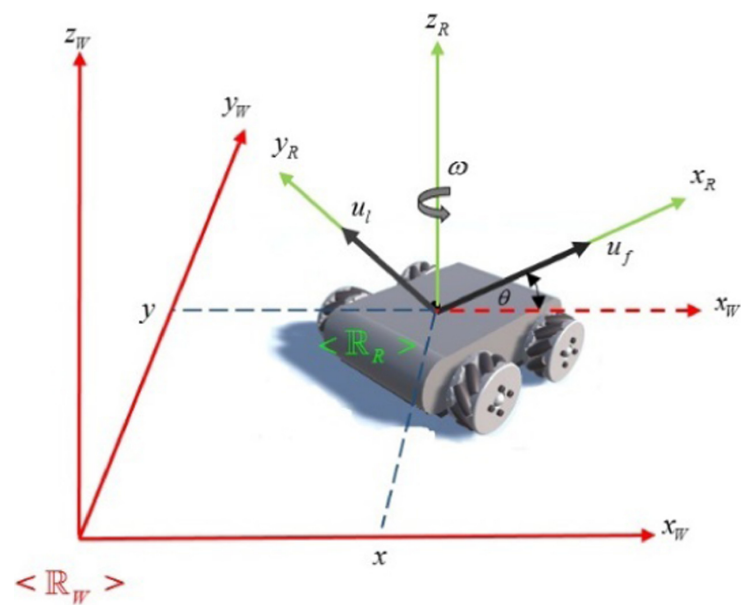

Fig. 1. Reference frames for omnidirectional robotic platform movement.

The omnidirectional platform consists of two linear speeds: Front $u_{f}$ and sideways $u_{l}$ found on the axes of the mobile reference system $<\mathbb{R}_{R}>$; in addition to an angular velocity $\omega$ that rotates around the axis $Z_{R}$. Rotation matrices are defined $\mathbf{R}_{W}^{R}(\theta)$ that rotates from the Robot frame of reference $<\mathbb{R}_{R}>$ to the inertial frame of reference $<\mathbb{R}_{W}>$ and $\mathbf{R}_{W}^{R}(\theta)$ that rotates from the inertial frame to the Robot frame, the latter is used to know the speeds of the mobile platform seen from the robot frame and represented by $\boldsymbol{\eta}$ performing the following process:

$$
\mathbf{R}_{W}^{R}(\theta)=\left[\begin{array}{lll}
\cos \theta & \sin \theta & 0 \\
-\sin \theta & \cos \theta & 0 \\
0 & 0 & 1
\end{array}\right] \quad ; \mathbf{R}_{R}^{W}(\theta)=\left[\begin{array}{lll}
\cos \theta & -\sin \theta & 0 \\
\sin \theta & \cos \theta & 0 \\
0 & 0 & 1
\end{array}\right] \boldsymbol{\eta}(t)=\left[\begin{array}{c}
u_{f} \\
u_{l} \\
\omega
\end{array}\right]=\mathbf{R}_{W}^{R}(\theta) \dot{\xi}(t)
$$


Obtained as a result the kinematic model that describes the movement of the omnidirectional robot with respect to the inertial reference system.

$$
\left\{\begin{array}{l}
\dot{x}=u_{f} \cos (\theta)-u_{l} \sin (\theta) \\
\dot{y}=u_{f} \sin (\theta)+u_{l} \cos (\theta) \\
\dot{\theta}=\omega
\end{array}\right.
$$

Where, $\dot{\xi}$ represents the derivative of the robot's positions relative to the inertial reference system $<\mathbb{R}_{W}>$ with $\xi=[x y \theta]^{T}$ and its variation over time $\dot{\xi}=[\dot{x} \dot{y} \dot{\theta}]^{T}$; also, is defined $\mathbf{W}=\left[W_{1} W_{2} W_{3} W_{4}\right]^{T}$ as the vector of the angular speeds of each wheel of the omnidirectional robotic platform that is obtained from:

$$
\mathbf{W}(t)=\mathbf{E}(a, b) \boldsymbol{\eta}(t)
$$

Considering that $\mathbf{E}$ is a constant transformation matrix that contains the parameters detailed below (see Fig. 2).

$$
\mathbf{E}=\frac{1}{r}\left[\begin{array}{lll}
1 & 1 & (a+b) \\
1 & -1 & -(a+b) \\
1 & 1 & -(a+b) \\
1 & -1 & (a+b)
\end{array}\right]
$$
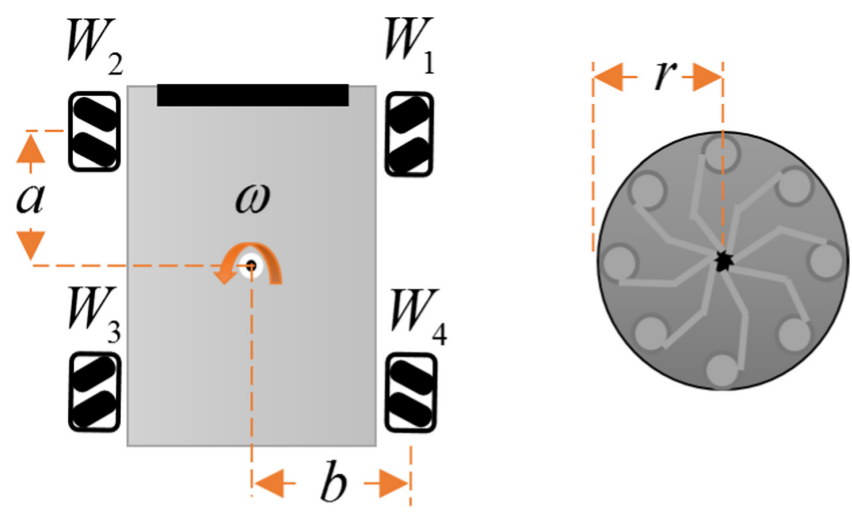

Fig. 2. Omnidirectional robotic platform parameters.

\subsection{Dynamic Model}

The dynamic model of the omnidirectional robotic platform is obtained from the EulerLagrange formulation, with the balance between the kinetic energy and potential of (4) to apply (5), where $\tau_{i}$ represents the generalized forces of the torques applied by the motors on each wheel. 


$$
\begin{gathered}
L=E_{C}-E_{P} \\
\tau_{i}=\frac{d}{d t}\left(\frac{\partial L}{\partial \dot{q}}\right)-\frac{\partial L}{\partial q} \\
\mathbf{E}_{\mathbf{C}}=\dot{\xi}^{T} \mathbf{M}_{R 1} \dot{\xi}+\mathbf{W}^{T} \mathbf{I}_{1} \mathbf{W}
\end{gathered}
$$

The sum of the robot's kinetic energies is represented by (6) where $M_{R 1}=$ $\frac{1}{2} \operatorname{diag}\left\{m_{R}, m_{R}, I_{R}\right\}$ is made up of the total mass of the robotic platform, $m_{R}$; the total inertia of the robotic platform, $I_{R}$; and the matrix $I_{1}=\frac{1}{2} \operatorname{diag}\left\{I_{W}, I_{W}, I_{W}, I_{W}\right\}$ containing the inertia of the wheels, $I_{\varphi}$. In addition, potential energy $E_{P}$ of the system is equal to zero, therefore developing (6) results (7) and when applying (5) is represented compactly in (8):

$$
\begin{gathered}
\frac{1}{2}\left(m_{R} \dot{x}^{2}+m_{R} \dot{y}^{2}+I_{R} \dot{\theta}^{2}+I_{W} W_{1}^{2}+I_{W} W_{2}^{2}+I_{W} W_{3}^{2}+I_{W} W_{4}^{2}\right)=\mathbf{E}^{T} \tau_{i} \\
\mathbf{R}_{W}^{R}(\theta)\left[\mathbf{E}_{\mathbf{C}}\right]_{\xi}+\mathbf{E}^{T}\left[\mathbf{E}_{\mathbf{C}}\right]_{\varphi}=\mathbf{E}^{T} \tau_{i} \\
{\left[\mathbf{E}_{\mathbf{C}}\right]_{\xi}=\mathbf{M}_{R 2} \ddot{\xi},\left[\mathbf{E}_{\mathbf{C}}\right]_{\varphi}=\mathbf{I}_{2} \dot{\mathbf{W}}}
\end{gathered}
$$

Then by replacing (9) in (8) results:

$$
\mathbf{R}_{W}^{R}(\theta) \mathbf{M}_{R 2} \ddot{\xi}+\mathbf{E}^{T} \mathbf{I}_{2} \dot{\mathbf{W}}=\mathbf{E}^{T} \tau_{i}
$$

In (10) the accelerations of the frame of reference $<\mathbb{R}_{W}>$ to the frame $<\mathbb{R}_{R}>$ are defined by $\ddot{\xi}=\mathbf{R}_{R}^{W}(\theta) \dot{\boldsymbol{\eta}}+\mathbf{R}_{W}^{R}(\theta) \boldsymbol{\eta}$; the accelerations of each wheel $\dot{\mathbf{W}}=\boldsymbol{E} \dot{\boldsymbol{\eta}}$; the matrices $\mathbf{M}_{R 2}=2 \mathbf{M}_{R 1}$ e $\mathbf{I}_{2}=2 \mathbf{I}_{1}$. So, the dynamic model of the omnidirectional robotics platform is represented as follows, where $\overline{\mathbf{M}}$ represents the mass matrix and $\overline{\mathbf{C}}$ is the matrix of centrifugal forces:

$$
\overline{\boldsymbol{M}} \dot{\boldsymbol{\eta}}+\overline{\boldsymbol{C}} \boldsymbol{\eta}=\mathbf{E}^{T} \tau_{i}
$$

However, since the control scheme proposed considers as input speeds and not torques, can define the behavior of the torque generated in each wheel,

$$
\tau_{i}=\frac{k_{p a}}{R_{p a}}\left(v_{i}-k_{p b} \mathbf{W}_{i}\right)
$$

in which have the different parameters of the equations of the dynamic model of a DC engine; $v_{i}$ as input voltages applied to each motor; $k_{p a}$ is the torque constant multiplied by the reduction constant; $R_{p a}$ is the electric resistance of the engine and $k_{p b}$ is the product of the constant reduction and the constant electromotive. The supply voltages $v_{v}=v_{u f}, v_{u l}, v_{\omega}$, in turn can be described based on a PD controller (13), where $\mathbf{K}_{P}$ is the matrix of proportional constants and $\mathbf{K}_{D}$ the matrix of derivative constants. 


$$
\mathbf{v}_{v}=\mathbf{K}_{P}\left(\boldsymbol{\eta}_{\text {ref }}-\boldsymbol{\eta}\right)-\dot{\boldsymbol{\eta}} K_{D}
$$

Through (10), (11), (12), (13) the compact mathematical model is obtained based on reference speeds and real speeds and accelerations:

$$
\begin{gathered}
{\left[\begin{array}{c}
u_{f r e f} \\
u_{\text {lref }} \\
\omega_{\text {ref }}
\end{array}\right]=\left[\begin{array}{lll}
\varsigma_{1} & 0 & 0 \\
0 & \varsigma_{2} & 0 \\
0 & 0 & \varsigma_{3}
\end{array}\right]\left[\begin{array}{c}
\dot{u}_{f} \\
\dot{u}_{l} \\
\dot{\omega}
\end{array}\right]+\left[\begin{array}{lll}
\varsigma_{4} & -\omega \varsigma_{5} & 0 \\
\omega \varsigma_{6} & \varsigma_{7} & 0 \\
0 & 0 & \varsigma_{8}
\end{array}\right]\left[\begin{array}{c}
u_{f} \\
u_{l} \\
\omega
\end{array}\right],} \\
\boldsymbol{\eta}_{r e f}(t)=\mathbf{M}(\varsigma) \dot{\boldsymbol{\eta}}(t)+\mathbf{C}(\varsigma, \boldsymbol{\eta}) \boldsymbol{\eta}(t),
\end{gathered}
$$

where $\varsigma \in \Re^{j}$ with $j=8$ and $\varsigma=\left[\varsigma_{1} \varsigma_{2} \ldots \varsigma_{j}\right]^{T}$ it is the vector of the dynamic parameters of the system and the matrices that represent the inertia of the omnidirectional robotic platform and the components of centripetal forces.

\section{Control Schemes}

The proposed control scheme for HIL simulation consists of two blocks, in the block "Control System" is the base nonlinear mimo controller or two cascading subsystems: a) Kinematic Controller, where position control errors are calculated at each measurement time and are used to drive the mobile robot in $a$ direction that decreases errors; and b) Dynamic Compensation, the objetive is to compensate for the dynamics of the omnidirectional robotic platform by reducing the speed tracking error. This controller receives as inputs the desired speeds calculated by the kinematic controller and to its output generates speed references that are sent through the communication channel. While the "Mathematical Model" block is the representation of the omnidirectional platform with its kinematic and dynamic mathematical model that returns the positions and speeds of the omnidirectional robotic platform (Fig. 3).

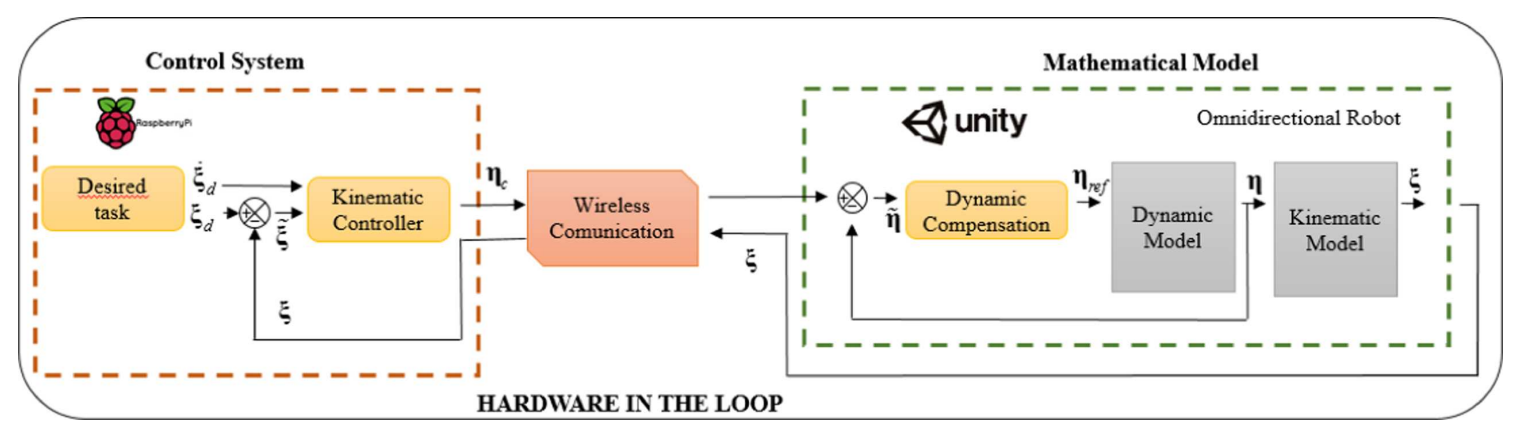

Fig. 3. Proposed HIL control scheme. 


\subsection{Designing the Control Algorithm}

The developed control algorithm is intended to perform desired path tracking tasks.

A) Kinematic Controller the objective of the proposed cinematic controller is to calculate the position error in each sampling period and use these values to move the omnidirectional robotic platform in the direction to reduce control errors. This kinematic controller is based on the kinematic model (2) and is represented as follows:

$$
\boldsymbol{\eta}_{c}=\mathbf{J}^{-1}\left(\dot{\xi}_{d}+\mathbf{G} \tanh (\tilde{\xi})\right)
$$

where, $\boldsymbol{\eta}_{c}$ represents the speeds of the kinematic controller; $\mathbf{J}^{-1}$ is the Jacobian matrix of the robotic platform; $\dot{\xi}_{d}=\left[\dot{x}_{d} \dot{\mathrm{y}}_{d} \dot{\theta}_{d}\right]^{T}$ is the derivative of the desired trajectory; $\mathbf{G}$ is the gain matrix to compensate for control errors; and $\tanh (\tilde{\xi})$ is the function of saturation of position errors contained in the matrix $\tilde{\xi}=\xi_{d}-\xi$.

In order to determine the stability of the controller, Lyapunov's candidate function of quadratic errors is determined $\mathbf{V}(\tilde{\xi})={ }_{1} \tilde{\xi}^{T}(t) \tilde{\xi}(t)$; and applying the time derivative, is obtain.

$$
\dot{\mathbf{V}}(\tilde{\xi})=\tilde{\xi}^{T}(t) \dot{\tilde{\xi}}(t)
$$

It is also defined (16) compactly considering speed errors $\xi(t)=\dot{\xi}_{d}(t)-\dot{\xi}(t)$ to get (18) and replace in (17)

$$
\begin{gathered}
\dot{\tilde{\xi}}(t)=-\mathbf{G} \tanh (\tilde{\xi}(t)) \\
\dot{\mathbf{V}}(\tilde{\xi})=-\tilde{\xi}^{T}(t) \mathbf{G} \tanh (\tilde{\xi}(t))
\end{gathered}
$$

Therefore, in order to ensure the stability of the proposed control law, it must be enforced that $\mathbf{G}>0$, ensuring that $\tilde{\xi}(t) \rightarrow 0$ when $t \rightarrow \infty$.

B) Dynamic Compensation, the objective of this block is to compensate for the dynamics of the omnidirectional robotic platform by reducing the speed tracking error. The desired speeds are input $\boldsymbol{\eta}_{c}$ and the output of this subsystem are reference speeds $\boldsymbol{\eta}_{r e f}$ to be sent to the robotic platform that in this case is located in the Unity $3 \mathrm{D}$ graphics engine.

$$
\boldsymbol{\eta}_{r e f}=\mathbf{M}\left(\dot{\boldsymbol{\eta}}_{c p}+\mathbf{K} \tanh (\tilde{\boldsymbol{\eta}})\right)+\boldsymbol{C} \boldsymbol{\eta}
$$

Considering that $\dot{\boldsymbol{\eta}}_{c p}$ represents those derived from the desired speeds; $\mathbf{K}$ is the gain matrix to compensate for control errors; and $\tilde{\boldsymbol{\eta}}$ is the vector of robotic platform speed errors. 
Similarly, for the stability analysis of the compensator, the candidate Lyapunov function of quadratic errors (in this case speeds) is determined.

$$
\begin{aligned}
& \mathbf{V}(\tilde{\boldsymbol{\eta}})={ }_{2}^{1} \tilde{\boldsymbol{\eta}}^{T}(t) \tilde{\boldsymbol{\eta}}(t) \\
& \dot{\mathbf{V}}(\tilde{\boldsymbol{\eta}})=\tilde{\boldsymbol{\eta}}^{T}(t) \dot{\tilde{\boldsymbol{\eta}}}(t)
\end{aligned}
$$

Working with the compact form (20) where acceleration errors are considered $\tilde{\boldsymbol{\eta}}=\dot{\boldsymbol{\eta}}_{c p}-\dot{\boldsymbol{\eta}}$, and by following the same procedure as in the previous case has:

$$
\begin{gathered}
\dot{\tilde{\boldsymbol{\eta}}}=-\mathbf{K} \tanh (\tilde{\boldsymbol{\eta}}) \\
\dot{\mathbf{V}}(\tilde{\boldsymbol{\eta}})=-\tilde{\boldsymbol{\eta}}^{T}(t) \mathbf{K} \tanh (\tilde{\boldsymbol{\eta}})
\end{gathered}
$$

Consolidating the stability of the proposed control law, when $\mathbf{K}>0$, ensuring that $\tilde{\boldsymbol{\eta}}(t) \rightarrow 0$ when $t \rightarrow \infty$.

\section{Experimental Results and Discussion}

This section presents the results of implementing the technique Hardware in the Loop, for which the construction of the omnidirectional robotic platform was carried out in order to evaluate the operation of the proposed controller, (see Fig. 4).

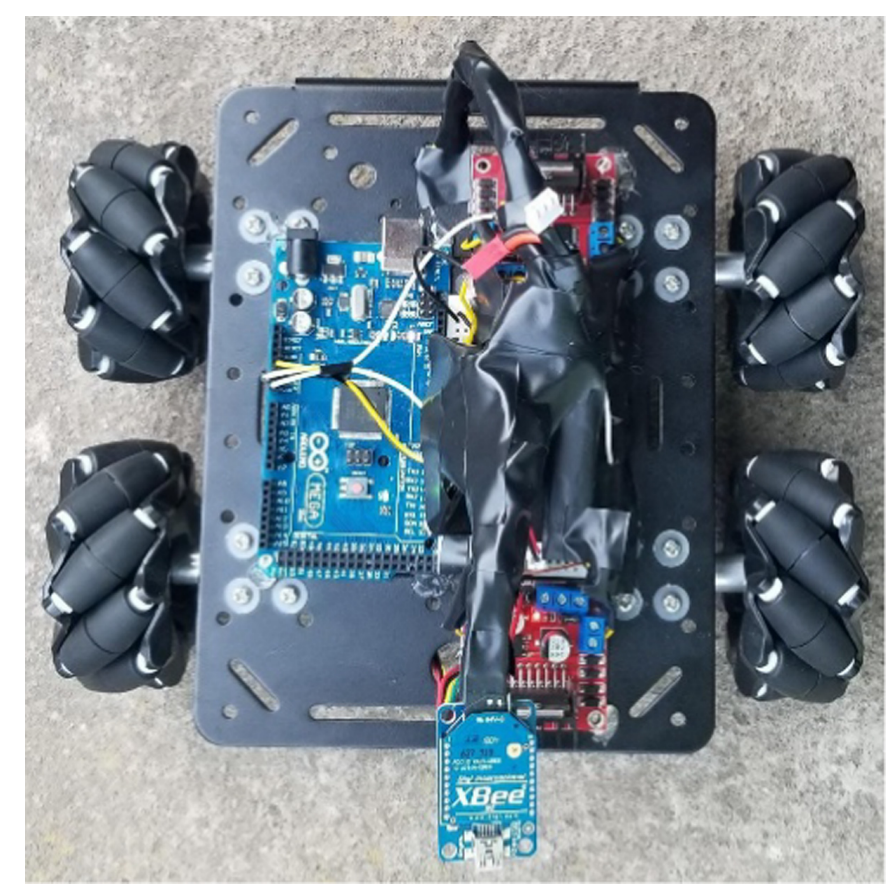

Fig. 4. Omnidirectional robotics platform built. 


\subsection{Omnidirectional Robot Construction}

This sub-section describes the construction of an omnidirectional mobile robot in which the hardware is divided into four modules, (see Fig. 5).

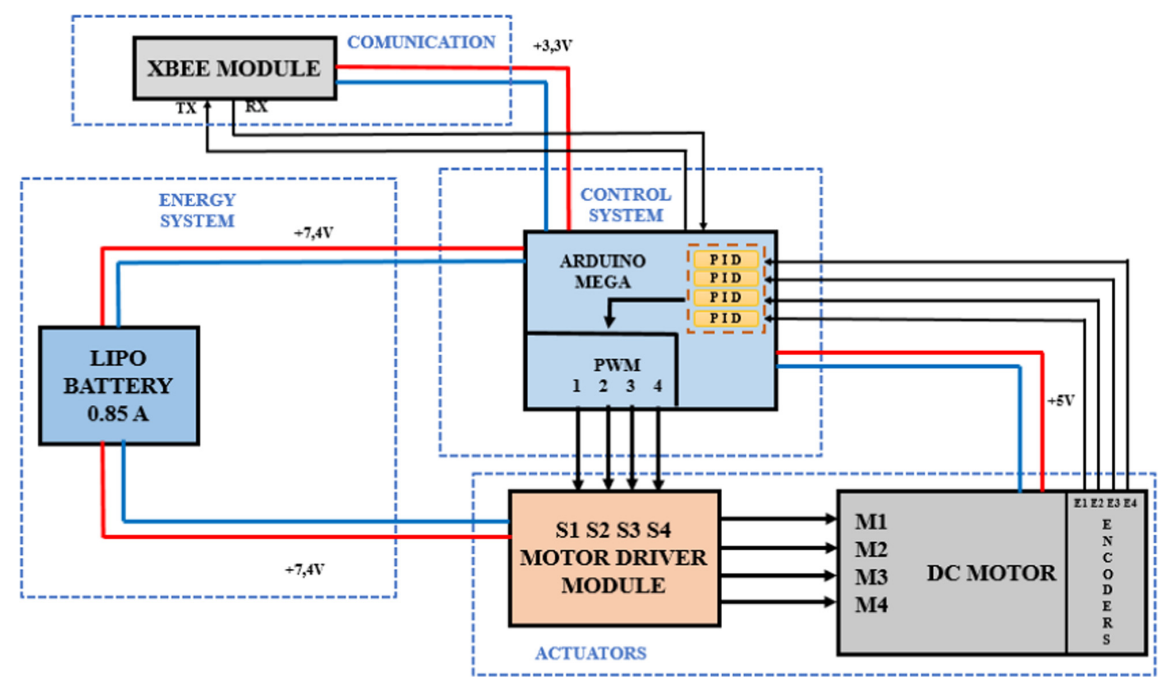

Fig. 5. Omnidirectional Robot Hardware Block Diagram.

Power System: Consists of a LIPO battery $(0.85 \mathrm{~A}$ and $7.4 \mathrm{v})$ that provides power to the entire system, directly connecting its rated voltage to the control module and actuator module; ii) Control system: This is composed of an Arduino Mega, which receives and transmits the signal of the kinematic control performed; in addition to compensate separately the dynamics of the mobile robot is implemented internal loop controllers (PIDs), compensating for the errors resulting from the subtraction between the desired speed and measured by each encoder located in the motors of the mecanum wheels.

Actuators: Consists of DC motors, with integrated speed sensors and controllers $(\mathrm{H}-$ bridge) on each motor that support a constant current of 2 A per channel.

Communication: Manages communication between the robot and the computer. When considering the transmission speed and distance of use of the mobile robot it was based on the IEEE 802.15.4 standard of Zigbee wireless networks.

\subsection{Identification and Validation}

The following expression is taken into account for the identification of dynamic model parameters:

$$
\mathbf{\Omega}(\boldsymbol{\eta}) \varsigma(t)=\boldsymbol{\eta}_{r e f}(t)
$$


By solving the operations of the matrices (20) and grouping the dynamic parameters, the linear parameterization in (26) required for implementation in the identification scheme (see Fig. 7).

$$
\left[\begin{array}{llllllll}
\dot{u}_{f} & 0 & 0 & u_{f} & \omega u_{l} & 0 & 0 & 0 \\
0 & \dot{u}_{l} & 0 & 0 & 0 & \omega u_{f} & u_{l} & 0 \\
0 & 0 & \dot{\omega} & 0 & 0 & 0 & 0 & \omega
\end{array}\right]\left[\begin{array}{c}
\varsigma_{1} \\
\varsigma_{2} \\
\varsigma_{3} \\
\vdots \\
\varsigma_{8}
\end{array}\right]=\left[\begin{array}{c}
u_{f \mathrm{ref}} \\
u_{l \text { ref }} \\
\omega_{\text {ref }}
\end{array}\right]
$$

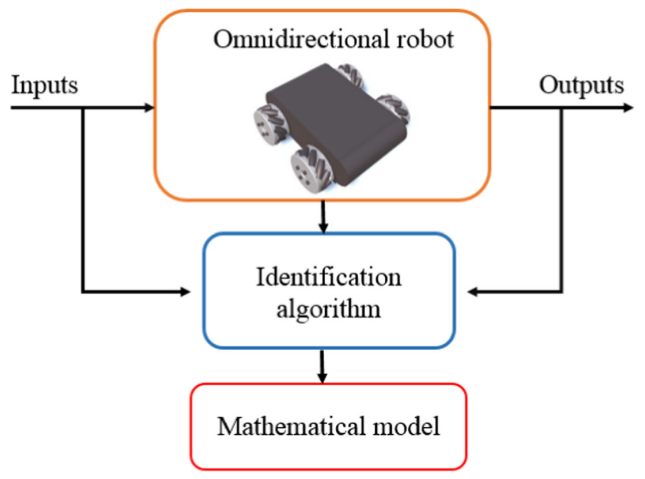

Fig. 7. Diagram for the identification of parameters of the Omnidirectional Robotic Platform.

Therefore, the identification of the parameters of the dynamic model of the robotic system was considered as excitation signals a series of steps of each speed to be fulfilled by the omnidirectional robotic platform $\boldsymbol{\eta}_{\text {ref }}$.
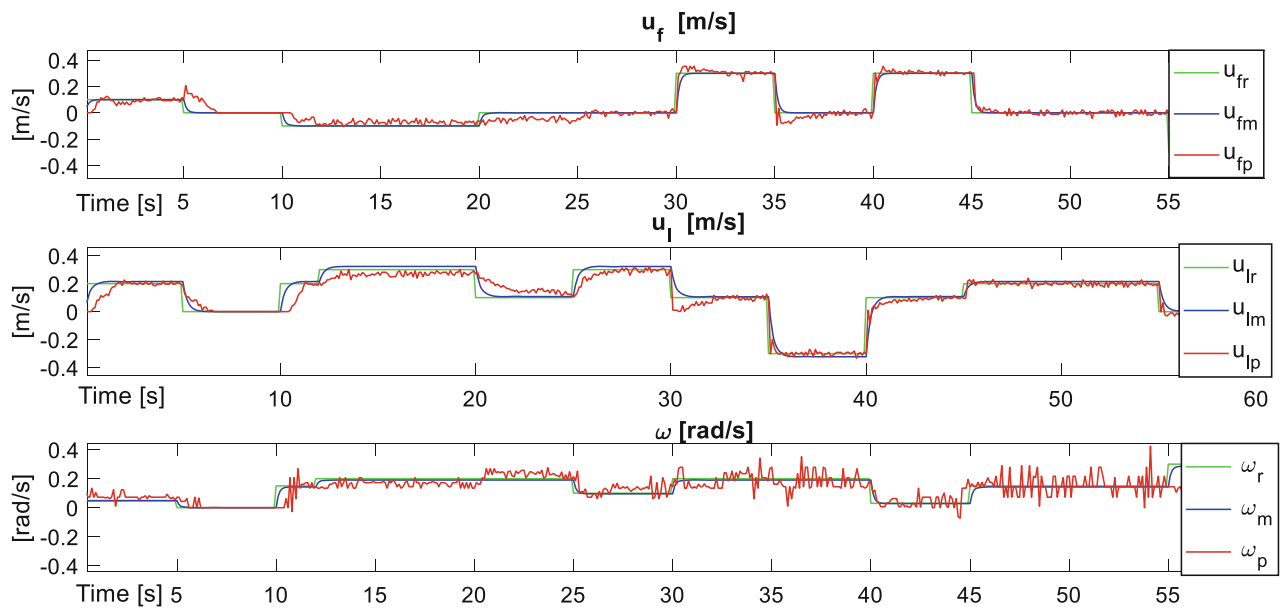

Fig. 8. Validation of dynamic parameters. 
To Fig. 8 shows the results obtained from the process of identification and validation of the calculated dynamic model, where $u_{f r}$ represents the desired reference front speed; $u_{f m}$ represents the front speed calculated by the model and $u_{f p}$ represents the real front speed obtained from the sensors of the robotic platform built, in the same way it is considered for lateral and angular speed. This data implements the least squares algorithm built into the schema (see Fig. 7) and the obtained parameters are as follows:

$\varsigma_{1}=0.3800, \varsigma_{2}=0.3800, \varsigma_{3}=0.1388, \varsigma_{4}=1.0744, \varsigma_{5}=-1.2639, \varsigma_{6}=1.2639$, $\varsigma_{7}=0.8749, \varsigma_{8}=1.2130$

\subsection{Implementation of HIL}

In the Fig. 9 shows the implementation of the HIL technique in a $3 \mathrm{D}$ virtual environment, in order to evaluate the performance of the control laws proposed for the omnidirectional robotic platform. The control laws are implemented in a Raspberry Pi 4 card for the autonomous trajectory tracking to be fulfilled by the robot; on the other hand, the kinematic and dynamic models are included in the Unity3D graphic engine to define the behavior of the robotic platform. All this is connected by means of wireless technology that allows to close the control loop.

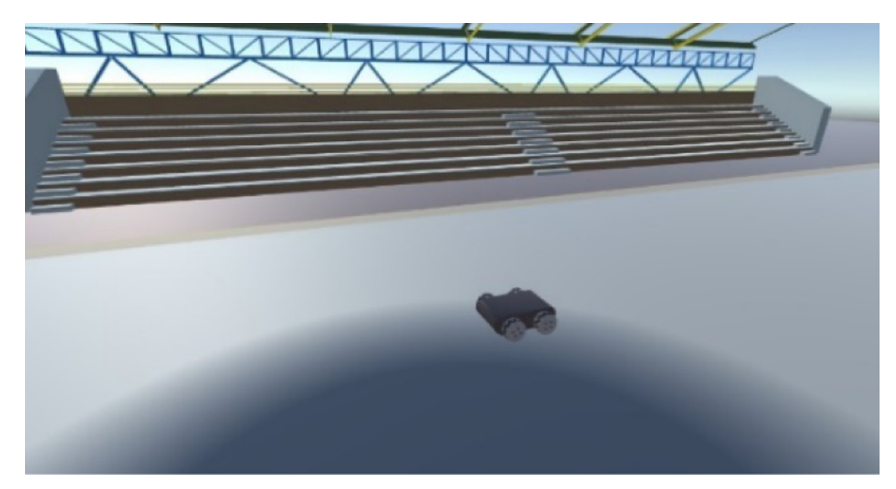

Fig. 9. Virtualized environment of the omnidirectional robotics platform.

For experimental testing, a circular trajectory was selected for compliance with both the robotic platform built, as well as the HIL scheme. 

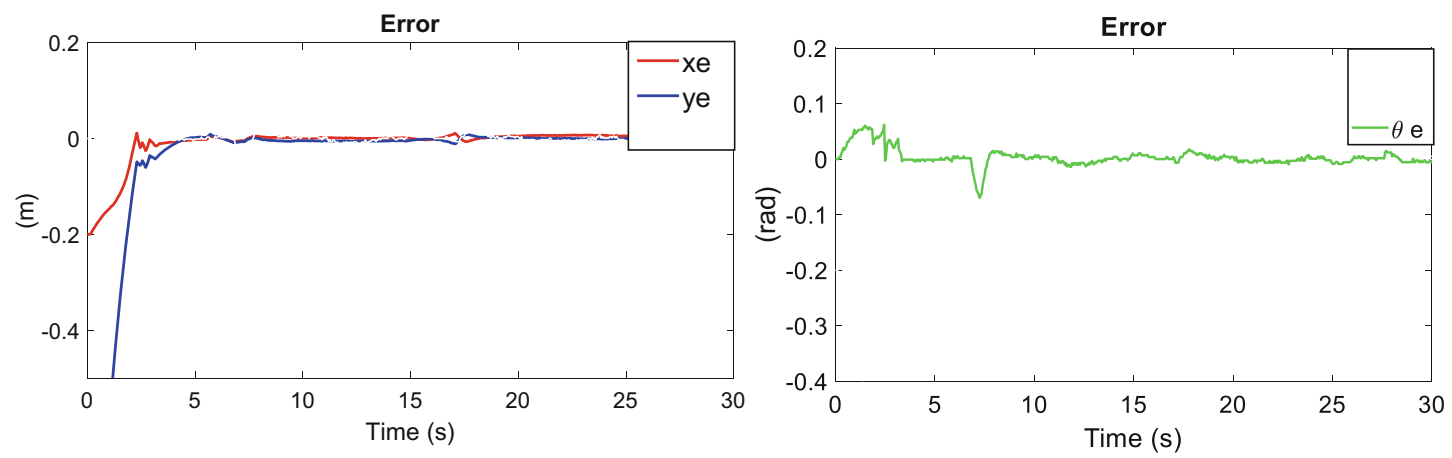

(a) Implemented in the robotic platform built
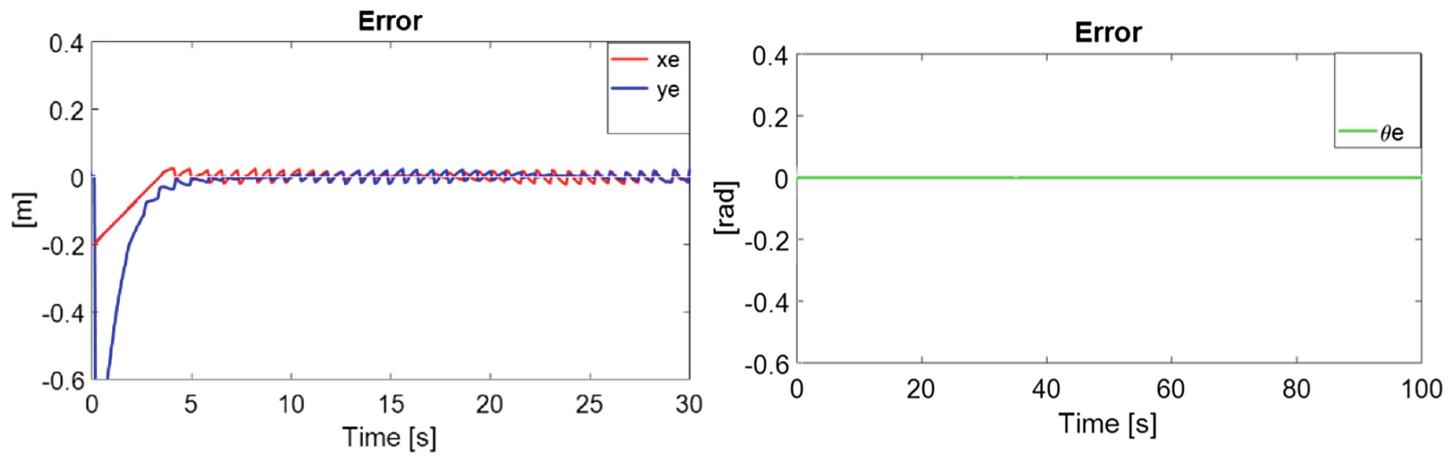

(b) Implemented with the HIL technique

Fig. 10. Autonomous path tracking control errors.

In the Fig. 10 is considered a sampling period of $100 \mathrm{~ms}$, which allows to calculate the position error at each moment and uses these values to choose the direction where these errors are reduced: $x e$ with respect to the $\mathrm{X}$ axis; $y e$ with respect to the $\mathrm{Y}$ axis; $\theta e$ with respect to the axis of the reference system $\left\langle\mathbb{R}_{W}>\right.$. In the same way that in control algorithms already implemented the errors tend to zero, however in this case when considering the dynamics of the robotic platform in the HIL technique it is observed that these are not fixed at zero, because it considers in a complete way the parameters that are going to interact with the robotic platform in a real operation.

\section{Conclusions}

The research carried out in different scientific databases determines that the construction of the omnidirectional robot with mecanum type wheels is more convenient to be carried out in $\mathrm{AB}$ configuration and the arrangement of the wheels should be rectangular. In addition, obtaining the kinematic model along with the dynamic model are used to improve the stability of the system which in turn provides greater accuracy of the behavior of the mathematical model with respect to the physical system, which allows to implement and evaluate advanced control algorithms considering as input signals the speeds of the robot, prior to the stability analysis of the proposed control scheme and validation of the dynamic model with the help of an identification algorithm in conjunction with the built robotic platform. This robot allows to implement 
control algorithms and at the same time to validate the HIL technique of the proposed scheme in the fulfillment of trajectories in an autonomous way. For which it is concluded that the technique HIL is a viable option to implement algorithms of control in situations in which it is not available the real plant, since it is possible to reproduce the behavior of the system of reliable form, which helps to the moment to evaluate these algorithms.

Acknowledgment. The authors would like to thank the Coorporación Ecuatoriana para el Desarrollo de la Investigación y Academia- CEDIA for their contribution in innovation, through the CEPRA projects, especially the CEPRA XIV-2020-08 project, "Tecnologias Inmersivas Multi-Usuario Orientadas a Sistemas Sinérgicos de Enseñanza-Aprendizaje"; also to the Universidad de las Fuerzas Armadas ESPE and the Research Group at ARSI, for the support for the development of this work.

\section{References}

1. Andaluz, V.H., Sásig, E.R., Chicaiza, W.D., Velasco, P.M.: Linear algebra applied to kinematic control of mobile manipulators. In: Kim, K.J., Kim, H., Baek, N. (eds.) ICITS 2017. LNEE, vol. 449, pp. 297-306. Springer, Singapore (2018). https://doi.org/10.1007/ 978-981-10-6451-7_35

2. Soliman, M., Azar, A.T., Saleh, M.A., Ammar, H.H.: Path planning control for 3-omni fighting robot using PID and fuzzy logic controller. In: Hassanien, A.E., Azar, A.T., Gaber, T., Bhatnagar, R., Tolba, M.F. (eds.) AMLTA 2019. AISC, vol. 921, pp. 442-452. Springer, Cham (2020). https://doi.org/10.1007/978-3-030-14118-9_45

3. Varela-Aldás, J., Andaluz, V.H., Chicaiza, F.A.: Modelling and control of a mobile manipulator for trajectory tracking. In: International Conference on Information Systems and Computer Science (INCISCOS), pp. 69-74 (2018)

4. Guo, P., Kim, H., Virani, N., Xu, J., Zhua M., Liu, P.: RoboADS: Anomaly detection against sensor and actuator misbehaviors in mobile robots. In: Annual IEEE/IFIP International Conference on Dependable Systems and Networks, pp. 574-585 (2018)

5. Karras, G.C., Fourlas, G.K.: Model predictive fault tolerant control for omni-directional mobile robots. J. Intell. Robot. Syst. 97, 635-655 (2019)

6. Khashayar, R. et al.: Vision-based integrated mobile robotic system for real-time applications in. Autom. Constr. 96, 470-482 (2018)

7. Wang, C., Liu, X., Yang, X., Hu, F., Jiang, A., Yang, C.: Trajectory tracking of an omnidirectional wheeled mobile robot using a model predictive control strategy. Appl. Sci. 8(2), 231-246 (2018)

8. Campos, J., Jaramillo, S., Morales, L., Camacho, O.: PSO tuning for fuzzy PD + I controller applied to a mobile robot trajectory control. In: International Conference on Information Systems and Computer Science (INCISCOS), pp. 62-68 (2018)

9. Vargas, M.F., Sarzosa, D.S., Andaluz, V.H.: Unified nonlinear control for car-like mobile robot 4 wheels steering. In: Mendes, A., Yan, Y., Chen, S. (eds.) Intelligent Robotics and Applications, pp. 182-194 (2018)

10. Valero, F., Rubio, F.: Assessment of the effect of energy consumption on trajectory improvement for a car-like robot. Cambridge University Press, pp. 1-12 (2019)

11. Sarmento, L., Nunes, F., Santos Martins, R., Sepúlveda, J., Sena Esteves, J.: Remote control system for a mobile platform with four Mecanum wheels. Int. J. Mech. Appl. Mech. 2017(1), 274-281 (2017) 
12. Andaluz, V.H., Carvajal, C.P., Arteaga, O., Pérez, J.A., Valencia, F.S., Solís, L.A.: Unified dynamic control of omnidirectional robots. In: Gao, Y., Fallah, S., Jin, Y., Lekakou, C. (eds.) TAROS 2017. LNCS (LNAI), vol. 10454, pp. 673-685. Springer, Cham (2017). https://doi. org/10.1007/978-3-319-64107-2_55

13. Shabalina, K., Sagitov, A., Magid, E.: Comparative analysis of mobile robot wheels design. In: 11th International Conference on Developments in eSystems Engineering (DeSE), pp. 175-179 (2018)

14. Andaluz, V.H., Carvajal, C.P., Santana, A.G., Zambrano, V.D., Pérez, J.A.: Navigation and dynamic control of omnidirectional platforms. In: Gao, Y., Fallah, S., Jin, Y., Lekakou, C. (eds.) TAROS 2017. LNCS (LNAI), vol. 10454, pp. 661-672. Springer, Cham (2017). https://doi.org/10.1007/978-3-319-64107-2_54

15. Andaluz, Víctor H., Molina, María F., Erazo, Yaritza P., Ortiz, Jessica S.: Numerical methods for cooperative control of double mobile manipulators. In: Huang, Y., Wu, H., Liu, H., Yin, Z. (eds.) ICIRA 2017. LNCS (LNAI), vol. 10463, pp. 889-898. Springer, Cham (2017). https://doi.org/10.1007/978-3-319-65292-4_77

16. Ortiz, J.S., Molina, M.F., Andaluz, V.H., Varela, J., Morales, V.: Coordinated control of a omnidirectional double mobile manipulator. In: Kim, K.J., Kim, H., Baek, N. (eds.) ICITS 2017. LNEE, vol. 449, pp. 278-286. Springer, Singapore (2018). https://doi.org/10.1007/ 978-981-10-6451-7_33

17. Saenz, E., Bugarin, V.S.: Kinematic and dynamic modeling of a four wheel omnidirectional mobile robot considering actuator dynamics. AMRob J. 114-120 (2016)

18. Andaluz, V.H., Arteaga, O., Carvajal, C.P., Zambrano, V.D.: Nonlinear control of omnidirectional mobile platforms. In: Huang, Y., Wu, H., Liu, H., Yin, Z. (eds.) ICIRA 2017. LNCS (LNAI), vol. 10464, pp. 354-364. Springer, Cham (2017). https://doi.org/10. 1007/978-3-319-65298-6_33

19. Joshi, A.: Real-Time Implementation and Validation for Automated Path Following Lateral Control Using Hardware-in-the-Loop (HIL) Simulation. SAE Technical Paper 2017-01-1683 (2017)

20. Shao, Y., Zulkefli, M.A.M., Sun, Z., Huang, P.: Evaluating connected and autonomous vehicles using a hardwarein-the-loop testbed and a living lab. Transp. Res. Part C 102, 121135 (2019)

21. Joshi, A.: Powertrain and Chassis Hardware-in-the-Loop (HIL) Simulation of Autonomous Vehicle Platform. SAE Technical Paper 2017-01-1991 (2017) 